\title{
A System to Support Laparoscopic Surgery by Augmented Reality Visualization
}

\author{
Stijn De Buck ${ }^{1}$, Johan Van Cleynenbreugel ${ }^{1}$, Indra Geys ${ }^{1}$, Thomas Koninckx ${ }^{1}$, \\ Philippe R. Koninck ${ }^{2}$, and Paul Suetens ${ }^{1}$ \\ 1 Faculties of Medicine and Engineering \\ Medical Image Computing (ESAT and Radiology) \\ 2 Department of Gyneacology \\ University Hospital Gasthuisberg \\ Herestraat 49 \\ B-3000 Leuven \\ stijn.debuck@uz.kuleuven.ac.be
}

\begin{abstract}
This paper describes the development of an augmented reality system for intra-operative laparoscopic surgery support.

The goal of this system is to reveal structures, otherwise hidden within the laparoscope view. To allow flexible movement of the laparoscope we use optical tracking to track both patient and laparoscope.

The necessary calibration and registration procedures were developed and bundled where possible in order to facilitate integration in a current laparoscopic procedure. Care was taken to achieve high accuracy by including radial distortion components without compromising real time speed.

Finally a visual error assessment is performed, the usefulness is demonstrated within a test setup and some preliminary quantitative evaluation is done.
\end{abstract}

\section{Introduction}

A laparoscopic surgery consists of making tiny holes into the peritoneum through which subsequently a camera and surgical tools are inserted. The camera view is in fact the only view on the surgery scene. Because this viewpoint is constantly changing to meet the surgeons need and because it is very different from the exoscopic view of the surgeon, the latter has to be very well trained and be able to interpret the laparoscopic images well. Due to its minimal invasive approach, laparoscopic surgery is able to reduce both the time of surgery and the recovery time of a patient.

The laparoscopic view though does not reveal all the structures the surgeon needs to see in order to complete the surgical procedure with success. These structures can for instance be hidden behind the peritoneal wall, like the ureter. This limitation can not only lead to a less efficiently performed surgery but also to further complications for the patient.

Often such structures can be extracted from pre-operative CT/MR-images. However the surgeon needs to interpret and to fuse these images mentally with 
the laparoscopic view. To alleviate this problem we propose an augmented reality approach in which the pre-operative images or at least the structures of interest deduced from these are visualized within the laparoscopic view. In this paper we describe the development of such a system and show our initial testing environment.

Previous work on augmented reality in the context of laparoscopic/endoscopic surgery has been concentrating on extracting 3D depth information from several images taken from different intra-operative positions. The resulting information can than be used to measure intra-operatively or display the $3 \mathrm{D}$ information over the endoscopic images [1]. Another application constructs a view of the intra-operative scene similar to conventional laparotomy [2]. Although these approaches also use an optical tracker for system and patient registration, they differ in a number of aspects. We augment the laparoscopic view, with which the surgeons are acquainted already. Since our approach can display structures totally invisible in the laparoscopic view, we believe it can be more versatile and holds potential to provide a considerable amount of added value to the surgeon.

\section{System Overview}

We use an optical tracker (Flashpoint 5000 of Image Guided Technologies), a laparoscope (Storz9050), and an Octane workstation (SGI) with Octane video card.

The tracker computes position and orientation of both patient and laparoscopic camera. In this way the flexibility the surgeon experiences today and which is necessary for proper navigation is preserved, while still providing an integrated visualization at real time rates.

The integrated visualization of virtual pre-operative objects, extracted from CT/MR-images, and the laparoscope view implies an accurate calibration of optical camera parameters. This algorithm should be as transparent as possible to the surgeon.

The combination of camera, optical tracker, together with pre-operative images requires registration procedures between at least three coordinate systems. Finally, visualization of integrated images should be realized at real time rates.

In the following we discuss the issues of calibration, registration and visualization more thoroughly.

\subsection{Camera Calibration and Registration}

We treat the camera calibration and registration problems together since they are closely related. The solution we propose integrates both thereby reducing the number of registration/calibration steps necessary to perform before surgery.

We attach a coordinate system to the laparoscopic camera $\left(C_{c}\right)$, one to the tracker $\left(C_{t}\right)$ and one to the pre-operative images $\left(C_{i}\right)$. If we register $C_{c}$ and $C_{t}$-which can be done pre-operatively-, only one patient-dependent registration operation has to be performed. Next, we show how to combine the camera calibration step and the registration of $C_{c}$ and $C_{t}$. 
Camera Calibration and $\boldsymbol{C}_{\boldsymbol{c}}-\boldsymbol{C}_{\boldsymbol{t}}$ Registration As shown by [1] the calibration of a laparoscopic/endoscopic camera demands a camera model that incorporates radial distortion. The calibration procedure by Tsai [3] satisfies this condition. Therefore we apply this procedure through the free-ware implementation of Willson [4]. In this model projection can be described mathematically as:

$$
w_{i}=K\left(R^{t} W_{i}+T\right)
$$

where $w_{i}$ is an image point $\left[\mu u_{i} \mu v_{i} \mu\right]^{t}, W_{i}$ a $3 \mathrm{D}$ point, $K$ an upper triangle projection matrix, $R$ a rotation matrix and $T$ a translation vector. The radial distortion in the images is modeled by:

$$
\begin{array}{r}
{\left[\begin{array}{l}
u_{i}^{(r d)} \\
v_{i}^{(r d)}
\end{array}\right]+\left[\begin{array}{l}
\delta u_{i}^{(r)} \\
\delta v_{i}^{(r)}
\end{array}\right]=\left[\begin{array}{l}
u_{i} \\
v_{i}
\end{array}\right]} \\
{\left[\begin{array}{l}
\delta u_{i}^{(r)} \\
\delta v_{i}^{(r)}
\end{array}\right]=\left[\begin{array}{l}
u_{i}^{(r d)}\left(k_{1} r_{i}^{2}+k_{2} r_{i}^{4}+\ldots\right) \\
v_{i}^{(r d)}\left(k_{1} r_{i}^{2}+k_{2} r_{i}^{4}+\ldots\right)
\end{array}\right]}
\end{array}
$$

with

$$
\begin{aligned}
& r_{i}=\sqrt{u_{i}^{2}+v_{i}^{2}} \\
& k_{1}, k_{2}=\text { the radial distortion coefficients } \\
& u_{i}^{(r d)}=\text { the radial distorted image x-coordinate } \\
& v_{i}^{(r d)}=\text { the radial distorted image y-coordinate }
\end{aligned}
$$

In order to automate the calibration, we designed a new calibration jig (cfr fig 1). The calibration procedure consists of three steps:

- First an endoscope image is taken by our camera of the calibration jig. The discs on the jig will project as ellipses in the image. The centroids of these ellipses can be extracted by iteratively applying the method described in [5] and taking into account the radial distortion. This results in a $2 \mathrm{D}$ image coordinate for each disc.

- Next, we correspond these 2D coordinates to their 3D locations. By attaching LED's to the calibration jig, we can compute the $3 \mathrm{D}$ coordinates of the black discs with respect to the $C_{t}$ reference frame. This way an extra $C_{c}-C_{t}$ registration step is made obsolete. A detailed description of the correspondence algorithm can also be found in [5].

- Finally these 2D-3D pairs are fed to the calibration algorithm of Tsai which returns the camera's intrinsic and extrinsic parameters.

Since in laparoscopic surgery the lens is changed before each surgery, it is an advantage for ergonomic reasons to combine both registration and optical camera calibration.

Tracker-Pre-operative Image Registration The goal of this procedure is to determine the $C_{t}-C_{i}$ relation. Markers are attached to the patient, which are visible in the pre-operative images. The same markers are indicated on the patient before surgery. Next, the 2 point sets are registered by means of the method of Arun et al. [6]. 


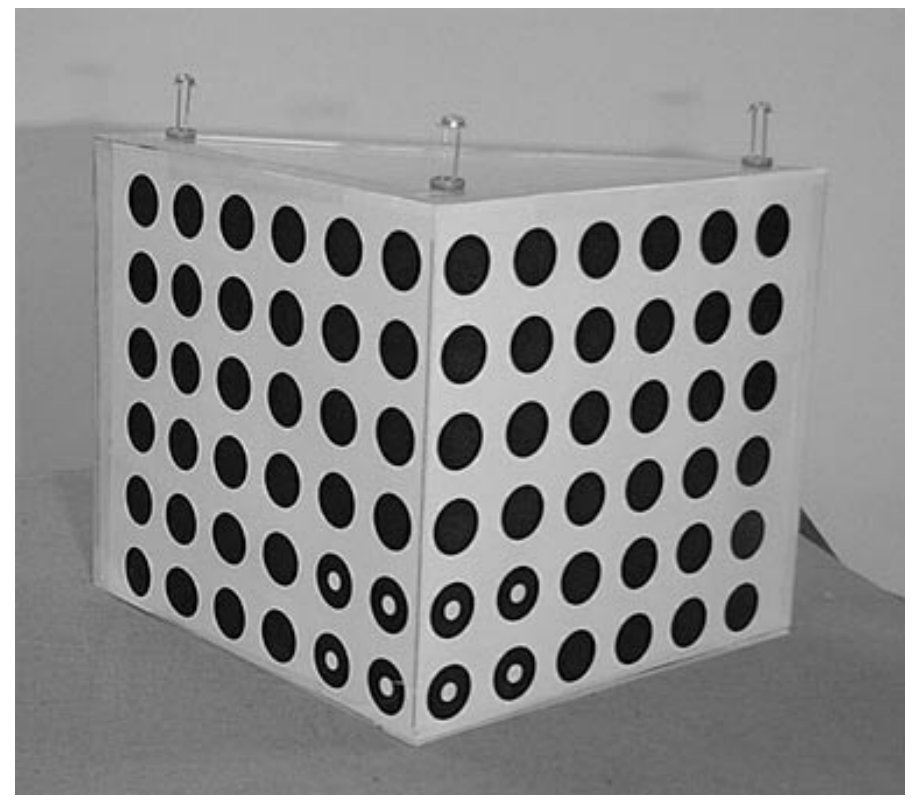

Fig. 1. The calibration/registration object by which the optical calibration and the registration of the laparoscope in the reference frame of the optical tracker are performed.

\subsection{Visualization}

In this section we treat two main issues in constructing an integrated visualization, namely:

- Visualizing the radial distortion at real time rate.

- Rendering the augmented view in a stable way.

Visualizing the Radial Distortion The result of our calibration are the extrinsic and intrinsic parameters describing how the laparoscopic camera perceives the world. Apart from the radial distortion component $k_{1}$, all the projection effects of the camera can be simulated by the pinhole model commonly used in computer graphics.

Implementing the radial distortion in the projection of the virtual objects would slow down the rendering [7, which in turn can compromise the real-time visualization constraint. In the case of laparoscopic surgery radial distortion can not be neglected (cfr section 2.1).

Therefore we propose a solution to render integrated images by rectification or de-distortion of the laparoscopic images. This is done by loading the laparoscopic video frames immediately into texture memory and warping this texture over a nurbssurface. The latter models the inverse radial distortion. A real-time visualization is obtained this way. 
Rendering the Pre-operative Images Once the real laparoscope images are rectified, the virtual objects can be rendered by the standard pinhole model. Virtual objects are extracted from pre-operative images by segmentation and surface extraction. Their position and orientation is modified by the position of the probe attached to the patient. Also the position and orientation of the virtual laparoscope is changed by the probe attached to the real one. The data coming from the tracker is filtered first in order to reduce the noise present in the measurement.

\section{A Setup for Error Evaluation}

To evaluate our system qualitatively, we have constructed a setup (cfr fig 2) to visually inspect the accuracy of calibration/registration and the efficiency of our visualization .

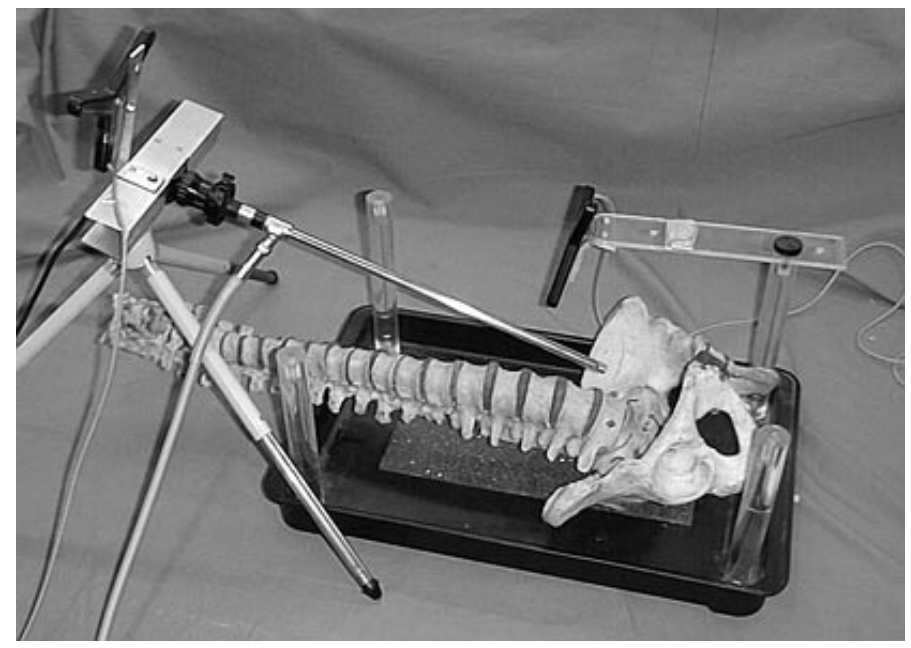

Fig. 2. The test setup we used to evaluate the system. Both camera and phantom are tracked.

The patient under surgery is represented by a phantom pelvis. To this phantom a number of radio-opaque markers were glued. The phantom was CTscanned and a surface model was generated. After calibration and registration, we can verify the accuracy by comparing the alignment of our model with the real images when using our system (cfr figure 3 en 44).

To illustrate the clinical usefulness a copper wire was attached to the phantom (before CT-scan), representing the ureter. In real laparoscopic surgery locating the ureter is a common problem. Under the assumption the ureter is not moved or deformed with respect to the pelvis, this problem can be solved by the system 


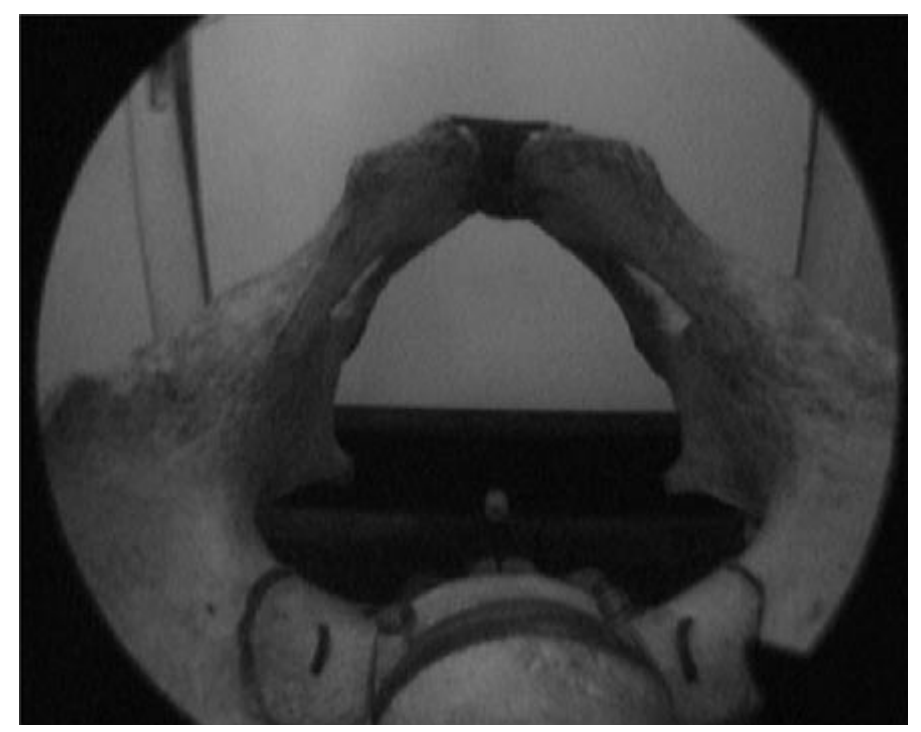

Fig. 3. A rectified laparoscope view constructed with the test setup

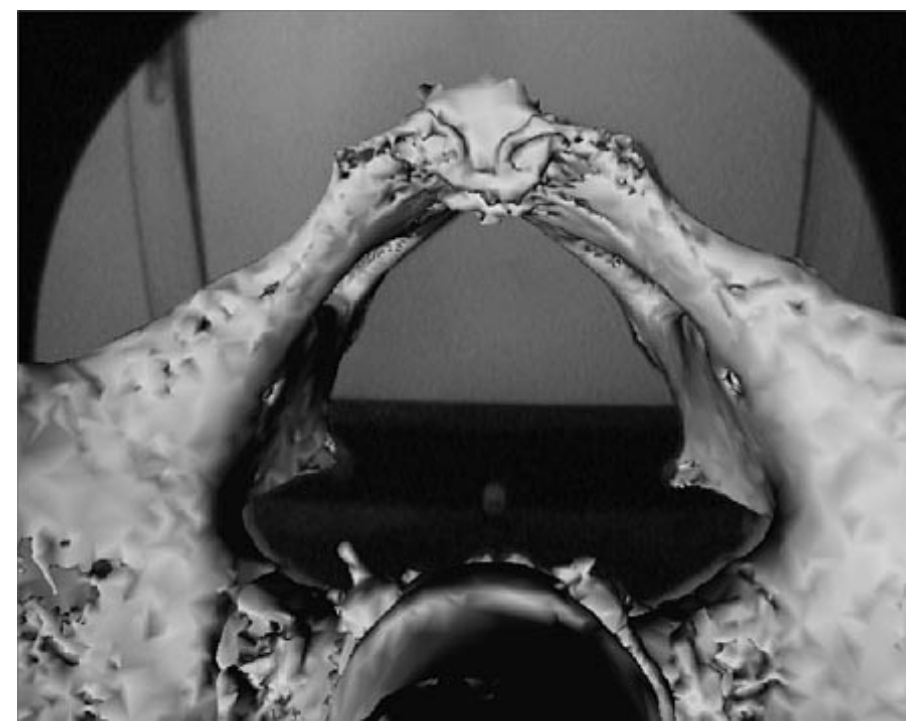

Fig. 4. Evaluation of the system by visual inspection 
by generating an integrated view of the real phantom (the surgery view) and of the segmented ureter (the pre-operative images) as in figure 5

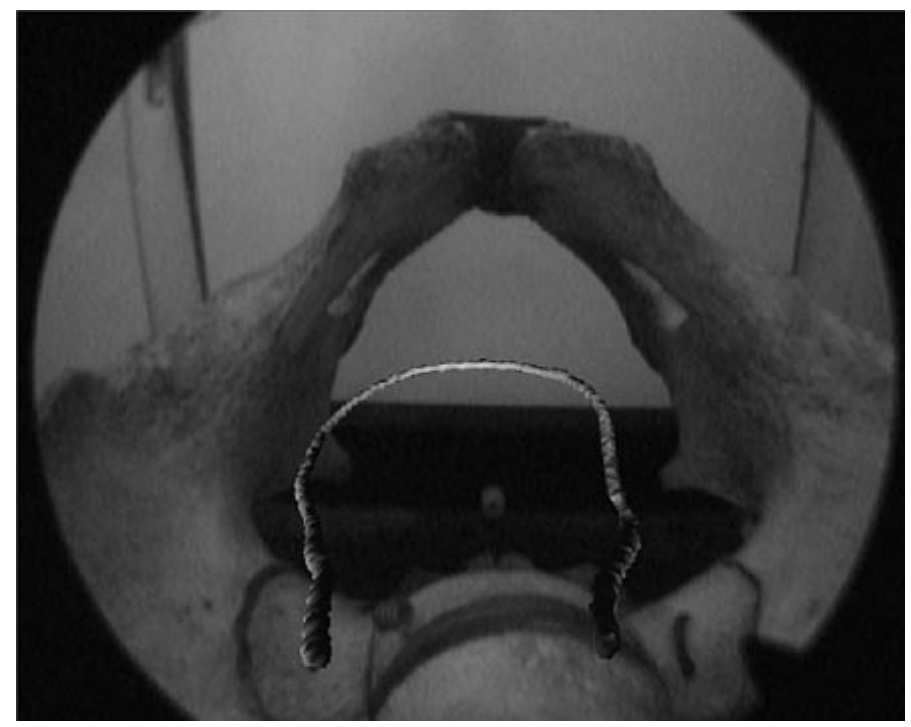

Fig. 5. Illustration of a possible application: although the ureter is invisible in the real view, it can be shown by augmented visualization.

We also include some preliminary quantitative evaluation results here. To assess a measure for the accuracy of the system as a whole, we displaced the calibration jig with respect to the calibration position. We than used our system to create a view without virtual objects and computed the image coordinates of the discs on the jig. Next, we compared these coordinates with the ones detected in the view. This resulted in an error ranging from 3.07 to 4.9 pixels for a displacement of 0 to $5 \mathrm{~cm}$. Since the displacement is not very large and the camera position did not change after calibration, more extensive testing has to be done here.

\section{Discussion}

In this paper we presented a system for intra-operative laparoscopic surgery support by augmented reality visualization. This system holds a large potential in gynaecological laparoscopy.

Several subproblems were solved which arise in a typical laparoscopic environment. First of all, a single calibration procedure was developed to solve both the optical calibration of the laparoscope and its registration with respect to the optical tracker. Second, a real time visualization method was implemented, 
taking into account the radial distortion of the laparoscope. Finally, tracker data was filtered and a stable augmented view was created.

We have shown a good qualitative evaluation within a test setup. Under the assumption of rigid movement of the hidden structures like the ureter -which will have to be validated-, we demonstrated the clinical usefulness of the system. We also showed some preliminary quantitative results which indicate an error of \pm 4 pixels.

Since no more than two extra procedures are necessary to use the system, we believe it can be easily integrated into a laparoscopic surgical procedure.

Our current work concentrates on further numerical quantification for the registration, calibration and tracking errors in the system.

\section{Acknowledgements}

This work is part of SuperVisie, ITA-II/980302, an IWT ITA-II project sponsored by the Flemish Government.

We also like to thank Storz for providing the laparoscope.

\section{References}

1. W. Konen, M. Scholz, and S. Tombrock. The VN project: endoscopic image processing for neurosurgery. Computer Aided Surgery, 3(3):145-148, December 1998.

2. H. Fuchs, M.A. Livingston, R. Raskar, D. Colucci, K. Keller, A. State, J.R. Crawford, P. Rademacher, S.H. Drake, and M.H. Meyer. Augmented reality visualization for laparoscopic surgery. In MICCAI, pages 934-943, October 1998.

3. R.Y. Tsai. An efficient and accurate camera calibration technique for 3D machine vision. In CVPR86, pages 364-374, 1986.

4. R.G. Willson. Freeware implementation of R. Tsai's camera calibration algorithm. http://www.cs.cmu.edu/afs/cs.cmu.edu/user/rgw/www/TsaiCode.html.

5. S. De Buck, J. Van Cleynenbreugel, G. Marchal, and P. Suetens. Towards visual matching as a way of transferring pre-operative surgery planning. In W. de Leeuw \& R. Van Liere, editor, Data Visualization 2000, pages 249-258, May 2000.

6. K.S. Arun, T.S. Huang, and S.D. Blostein. Least-squares fitting of two 3-d point sets. PAMI, 9(5):698-700, September 1987.

7. P.J. Edwards, A.P. King, C.R. Maurer Jr, D.A. de Cunha, D.J. Hawkes, D.L.G. Hill, R.P. Gaston, M.R. Fenlon, A. Jusczyzck, A.J. Strong, C.L. Chandler, and M.J. Gleeson. Design and evaluation of a system for microscope-assisted guided interventions (magi). IEEE Transactions on Medical Imaging, 19(11):1082-1093, November 2000. 